\title{
CONTAMINAÇÃO DE SUPLEMENTOS DIETÉTICOS USADOS PARA PRÁTICA ESPORTIVA:
}

\author{
UMA REVISÃO DE LITERATURA
}

\author{
ESP. RENATA MARQUES TOEWS PARRA \\ Especialista em Nutrição aplicada ao Esporte e Fitness Corporativo (UFRJ) \\ Clube de Regatas Flamengo (Rio de Janeiro - Rio de Janeiro - Brasil) \\ E-mail: renata_parra@yahoo.com.br \\ DR. ALEXANDRE PALMA \\ Doutor em Saúde Pública (ENSP/FIOCRUZ) \\ Universidade Federal do Rio de Janeiro (Rio de Janeiro - Rio de Janeiro - Brasil) \\ E-mail: palma_alexandre@yahoo.com.br \\ Dra. ANNA PAOLA TRINDADE ROCHA PIERUCCI \\ Doutora em Ciências de Alimentos (UFRJ) \\ Universidade Federal do Rio de Janeiro (Rio de Janeiro - Rio de Janeiro - Brasil) \\ E-mail: appierucci@gmail.com
}

\begin{abstract}
RESUMO
O objetivo do presente estudo foi revisar a literatura que trata da contaminação dos suplementos alimentares por substâncias consideradas nocivas à saúde ou doping para os atletas. Para tanto, foram revisados artigos científicos indexados nas bases de dados Medline e Scielo. Foi possível observar que algumas investigações detectaram doses excessivas de ingredientes potencialmente tóxicos, não indicados no rótulo e considerados doping. Entre as substâncias proibidas encontradas em alguns suplementos estão os esteróides anabólico-androgênicos e os psicoestimulantes. Neste sentido, é possível alegar que o uso de suplementos dietéticos por praticantes de atividades físico-esportivas, competitivas ou não, carece de maior segurança e deve ser realizado com muito critério.
\end{abstract}

PALAVRAS-CHAVE: Suplementos dietéticos; esteróides anabólico-androgênicos; contaminação; doping. 


\section{INTRODUÇÃO}

Suplemento dietético é uma expressão genérica que tem sido utilizada para designar qualquer substância ingerida de forma oral que contenha elementos com capacidade para complementar a dieta. Frequentemente comercializados sob a forma de comprimidos, líquidos, géis, pós ou barras, estas substâncias podem ser derivadas de plantas, vitaminas, aminoácidos, proteínas, minerais, carboidratos, entre outros aspectos. Além disso, também tem sido denominado de "suplemento nutricional", "suplemento esportivo" ou mesmo "ergogênico".

Em que pese as evidências científicas da eficácia do uso de suplementos dietéticos serem limitadas, o mercado global de suplementos, em 200 I, foi estimado em 46 bilhões de dólares. Nos Estados Unidos este mercado foi responsável por valores que superaram 16 bilhões de dólares no ano 2000 (MAUGHAN; KING; LEA, 2004). Maughan, King e Lea, (2004) ainda explicam que, embora boa parte seja utilizada por atletas de elevado rendimento, uma parcela importante é consumida pela população ativa, em geral.

Em pesquisa recente, conduzida por Braun et al. (2009) com atletas alemães de diferentes níveis, foi possível observar que atletas de maior nível apresentaram prevalência de 100\% para o uso de suplementos alimentares. Entre os atletas de menores níveis foram reportadas prevalências que variaram de 72 a $81 \%$.

Kristiansen et al. (2005), ao estudarem a prevalência do uso de suplementos dietéticos entre um grupo de atletas e outro de não atletas (grupo controle), ambos universitários canadenses, observaram que $98,6 \%$ dos atletas e 94,3\% do grupo controle manifestaram ter feito uso dos suplementos. Este estudo também apontou que os amigos estão entre os que mais frequentemente recomendam o uso; que grande parte das informações advém da Internet; e que existe pouco conhecimento destas substâncias pelos usuários (KRISTIANSEN et al., 2005).

Investigações semelhantes foram realizadas entre praticantes de exercícios físicos em academias de ginástica. Nos Estados Unidos, Morrison, Cizis e Shorter (2004) verificaram que a grande maioria (84,7\%) já havia feito uso de ao menos um suplemento alimentar. Deste grupo, 93,4\% reportaram fazer uso regular ao longo de todo ano, enquanto 5,5\% revelaram fazer uso somente em períodos específicos. No Brasil, Pereira, Lajolo e Hirschbruch (2003) identificaram taxa de prevalência expressivamente menor $(23,9 \%)$ entre os praticantes que consumiam ao menos um suplemento.

Por outro lado, uma análise de revistas populares, nos Estados Unidos, durante o período entre 1994 e 1998 revelou que há uma abundância de informes publicitários e reportagens sobre suplementos dietéticos, seja em artigos jornalísticos ou 
em propagandas. Contudo, mais de dois terços dos artigos não incluem informações completas que permitam o uso com segurança. Deste modo, os autores do estudo chamam a atenção que embora essas publicações contenham extensivas informações sobre suplementos dietéticos, elas não fornecem todas as informações necessárias sobre os riscos e o modo de uso seguro destas substâncias (KAVA et al., 2002).

Um dos riscos comumente evocados pelos pesquisadores do tema diz respeito à contaminação dos suplementos por substâncias que podem causar reações adversas ou problemas à saúde do consumidor e que, por vezes, no meio esportivo são consideradas doping (MAUGHAN; KING; LEA, 2004). Embora esta contaminação possa, em muitos casos, ser decorrente da manipulação inadequada, há evidências de que possa ser resultado da adulteração deliberada do produto (MAUGHAN, 2005). Considerando o modo de produção capitalista e a consequente lógica do lucro financeiro embutido nesta ideologia, não é arriscado pensar que as alterações nos suplementos estejam ligadas a vantagens comerciais. $\bigcirc$ suplemento, como um objeto transformado em mercadoria, deve cumprir suas "promessas de felicidade". E se o valor de uso de uma mercadoria está associado à sua utilidade, é preciso ponderar que somente aquilo que, no objeto, é fonte de comparação com outros objetos permite o processo de troca, isto é, atribui-lhe um valor de troca. Se esta relação fracassa, o objeto pode ser retirado de circulação. Deste modo, o suplemento necessita ser "eficaz" em levar o consumidor a alcançar seus objetivos (PIGNARRE, 1999).

Neste sentido, o presente estudo objetiva revisar a literatura que trata da contaminação dos suplementos alimentares por substâncias consideradas arriscadas à saúde ou doping para os atletas.

Para tanto, foram pesquisados artigos científicos indexados nas bases de dados medline e scielo a partir das palavras-chaves "suplementos dietéticos", "suplementos alimentares", "contaminação", "nutrição esportiva" e "doping".

\section{CONTAMINAÇÃO DOS SUPLEMENTOS ALIMENTARES}

De acordo com a World Anti-doping Agency - WADA (WORLD ANTI-DOPING AGENCY, 2003) tem sido considerado doping toda e qualquer conduta que viole as normas antidoping expressas nos artigos 2. I a 2.8 do código antidoping da Agência. Entre as diferentes infrações, se incluem a utilização ou tentativa de utilização de substâncias listadas como proibidas, bem como, a presença destas ou de seus metabólitos na amostra coletada por um esportista (WORLD ANTI-DOPING AGENCY, 2007). É interessante destacar, ainda, que o inciso 2. I . I do artigo 2. I do código da Agência Mundial Antidoping ressalta que é de responsabilidade pessoal de 
cada praticante assegurar o uso ou não de cada substância listada como proibida, independentemente da intenção ou negligência (WORLD ANTI-DOPING AGENCY, 2003). Neste sentido, o atleta poderá sofrer sanções mesmo que faça uso inocente das substâncias proibidas (Princípio da responsabilidade objetiva).

Por outro lado, a Agência Mundial Antidoping não proíbe, mas se posiciona sobre o uso dos suplementos dietéticos. Para a Agência (WORLD ANTIDOPING AGENCY, 2005), o uso de suplementos tem sido uma preocupação porque vários países não regulam a fabricação destes produtos, facilitando assim os casos em que os ingredientes apresentados nos rótulos não correspondem integralmente àqueles presentes no suplemento e, em alguns casos, algumas substâncias presentes podem ser proibidas pela regulamentação antidoping. Isto ocorre, provavelmente, porque as empresas produtoras dos suplementos não são obrigadas a declarar integralmente sua composição, além de não seguirem, em sua maioria, as normas técnicas ideais para produção de substâncias em laboratório, conhecidas internacionalmente como Good Manufactoring Practices (GMP) (WORLD ANTI-DOPING AGENCY, 2005).

Segundo Morrison, Cizis e Shorter (2004), embora alguns suplementos possam, de fato, ser benéficos, tem sido demonstrado que outros podem ser inócuos ou perigosos e advertem que muitos efeitos adversos decorrentes da utilização de alguns suplementos foram notificados à Food and Drug Administration (FDA), nos Estados Unidos. Van der Merwe e Grobbelaar (2005) corroboram estas afirmações e lembram que o uso indiscriminado de suplementos nutricionais é uma potencial causa do aumento de casos positivos de doping entre os atletas olímpicos de alto nível. Para os autores, existe no mercado uma imensa variedade de substâncias nutricionais específicas para atletas que podem ser utilizadas com os propósitos de melhorar o rendimento, facilitar o processo de recuperação, manipular a composição corporal e reduzir o mal estar e os riscos de infecções. E, como estão disponíveis sem prescrição e são vendidos legalmente, alguns esportistas presumem que esses produtos não contêm substâncias proibidas.

Instituições como a American Dietetic Association, Dietitians of Canada e American College of Sports Medicine têm sugerido que o uso de suplementos alimentares por praticantes de atividades físico-esportivas deveria ser feito com cautela e somente após cuidadosa avaliação, uma vez que, a despeito dos presumíveis efeitos favoráveis defendidos na literatura, o uso de suplementos dietéticos pode, ao mesmo tempo, provocar efeitos adversos (JUHN, 2003; AMERICAN DIETETIC ASSOCIATION; DIETITIANS OF CANADA; AMERICAN COLLEGE OF SPORTS MEDICINE, 2000). 
No Brasil, ainda existem poucos estudos sobre o uso de agentes ergogênicos pela população. Segundo Barros Neto (200I), há um uso excessivo de agentes ergogênicos farmacológicos, completamente em desacordo às necessidades nutricionais dos atletas e às evidências científicas publicadas na literatura.

Entre as investigações é possível identificar que muitos suplementos alimentares podem conter esteróides anabolizantes ou pré-hormônios, que são metabolizados e se transformam em esteróides anabólicos-androgênicos. Igualmente, também tem sido demonstrado que alguns suplementos podem conter outros tipos de substâncias proibidas, como os estimulantes. Neste sentido, o consumo de suplementos pode colocar em risco à saúde do consumidor.

\section{CONTAMINAÇÃO POR ESTERÓIDES ANABÓLICO-ANDROGÊNICOS OU PRÉ- HORMÔNIOS}

Os esteróides anabólicos têm sido considerados produtos químicos derivados do hormônio masculino testosterona (SANTOS, 2003). Por outro lado, o termo pré-hormônio refere-se a uma classe de substâncias hormonais precursoras da produção hormonal. Seu uso no meio esportivo, no entanto, é proibido, uma vez quer resultam em substâncias detectadas no exame anti-doping e o atleta sujeito às mesmas sanções relativas ao uso de esteróides anabólico-androgênicos (SANTOS, 2003). A androstenediona, o androstenediol, a dihidroandrostenediona (DHEA) e a dehidroepiandrosterona são considerados precursores de testosterona e, muitas vezes, são encontrados nos suplementos nutricionais (BOYCE, 2003; CHYKA, 2003).

Não é raro que os atletas se utilizem de recursos ergogênicos com o propósito de aumentar o rendimento e, assim, as chances de vitória em competições. Neste sentido, frequentemente esportistas têm buscado estes recursos e entre esses, a despeito do caráter moralmente questionável, dos efeitos adversos à saúde e das políticas proibitórias, estão os esteróides anabólicos-androgênicos. Segundo Silver (200 I), é estimado que de I a 3 milhões de norte-americanos, de ambos os sexos, já fizeram uso deliberado destas substâncias ao longo da vida.

Por outro lado, tem sido demonstrado que alguns suplementos considerados não hormonais, tais como, vitaminas, minerais e aminoácidos, podem conter substâncias hormonais, a despeito de não estarem declaradas ou listadas nos rótulos e serem, por vezes, incluídas como proibidas pela Agência Mundial Antidoping. Neste sentido, Geyer et al. (2004) encontraram que de um total de 634 suplementos pesquisados, 94 (14,8\%) continham pré-hormônios não listados no rótulo. Além disto, verificaram que os suplementos considerados positivos para o conteúdo de esteróides anabólico-androgênicos continham uma concentração que variou de 0.0 I 
$\mu \mathrm{g} / \mathrm{g}$ a $190 \mu \mathrm{g} / \mathrm{g}$. Este estudo, desenvolvido por pesquisadores do Laboratório de Colônia a pedido da Comissão Médica do Comitê Olímpico Internacional, revelou também que de todos os suplementos dados como positivos, 23 (24,5\%) continham compostos relacionados à nandrolona e à testosterona, $64(68,1 \%)$ continham pré-hormônios da testosterona e sete (7,5\%) pré-hormônios da nandrolona, cujos objetivos de uso são o aumento da massa muscular e da força. Ademais, 45 (47,9\%) suplementos continham mais de um esteróide e oito (8,5\%) a combinação de cinco ou mais diferentes compostos de esteróides (MAUGHAN, 2005).

Em investigação conduzida para identificar se a ingestão de suplementos nutricionais contaminados poderiam resultar positivo ao doping em cinco homens saudáveis, Van der Merwe e Grobbelaar (2005) observaram que os indivíduos ao ingerirem uma cápsula de suplemento dietético contendo | 9-nor-4-androstenediona, que não estava listado no rótulo do suplemento, apresentaram concentrações de 19-norandrosterona acima do limite aceito pela WADA em até 12 horas após a administração; a maior concentração de 19 -androstenediona foi de 54,6 ng/ml 8 horas após a administração; e, em dois voluntários os valores de 19 norandrosterona aceitos pela WADA foram superados até 36 horas após a administração. Deste modo, os resultados mostraram que o consumo de microgramas de substância proibida contida nos suplementos alimentares pode levar o atleta a apresentar resultados positivos no teste de doping.

Catlin et al. (2000) realizaram pesquisa semelhante com 4I homens com idade entre 20 e 44 anos com o objetivo de avaliar o conteúdo e a pureza dos suplementos com traços de androstenediona e 19-norandrosterona, vendidos de forma legal sem prescrição médica, além de verificar se a quantidade destas substâncias acarretariam em testes positivos ao doping. A amostra foi dividida em diferentes grupos. Um grupo ( $\mathrm{n}=13$ ) recebeu $100 \mathrm{mg} / \mathrm{d}$ em dose oral (cápsulas) de androstenediona; outro $(n=1$ I) recebeu $300 \mathrm{mg} / \mathrm{d}$ por sete dias; um terceiro grupo $(n=13)$ não recebeu nenhuma androstenediona e, por fim, a um último grupo $(n=4)$ foi administrado I O $\mu \mathrm{g}$ de 19-norandrostenediona. A pesquisa demonstrou que algumas marcas comercializadas apresentavam pré-hormônios não declarados nos rótulos. O estudo, ainda, sugere que traços de contaminação por 19-norandrosterona por quem consumiu androstenediona são suficientes para causar resultados positivos para o teste antidoping de urina. Por outro lado, doses orais de esteróides menores que I $0 \mu \mathrm{g}$ foram absorvidas e excretadas na urina.

Com o propósito de investigar a presença de 19-nor-4-androstena-3, 17diona e 4-androstena-3, 17-diona em um suplemento alimentar sob a forma de cápusla, De Cock et al. (200I) analisaram a urina coletada de cinco voluntários. Embora as razões androstenediona/epitestosterona e testosterona/epitestosterona 
não tenham apresentado diferenças significativas após a administração, concentrações acima de $2 \mathrm{ng} / \mathrm{ml}$ para norandrosterona, um importante metabólico da nadrolona, foram detectadas até 144 horas após a ingestão do suplemento alimentar.

Baume et al. (2006) analisaram a presença de estimulantes e de pré-hormônios na composição de 103 suplementos dietéticos vendidos pela internet e verificaram que três apresentaram grandes quantidades do esteróide anabólico metandienona na urina, o que seria considerado como um teste de antidoping positivo. Os resultados também mostraram que outros produtos continham traços de hormônios ou pré-hormônios não descritos nos rótulos. Os suplementos analisados foram divididos em quatro categorias: Creatina (creatina monoidratada); Pré-hormônios (DHEA, androstenediol, androstenediona, norandrostenediol e norandrostenediona); Aminoácidos (I-valina, I-leucina, I-isoleucina) e Psicoestimulantes (efedrina, cafeína, Ma Huang, guaraná, sinefrina). Os três primeiros consumidos com o propósito de contribuir à síntese protéica e ao aumento da massa muscular e da força. $\bigcirc$ último consumido para aumentar o metabolismo e acelerar a perda de gordura corporal.

A análise de 75 diferentes suplementos dietéticos vendidos na internet foi conduzida por Kamber et al. (200 I). Entre os principais achados os pesquisadores verificaram que sete de 17 suplementos classificados como pré-hormônios continham substâncias não indicadas nos rótulos, o que corresponde a $41 \%$ dos produtos desta classe e $9 \%$ de todos os suplementos analisados. Embora não tenham sido encontrados esteróides anabólicos-androgênicos nos suplementos investigados e, por isso, impossibilitada a demonstração de contaminação, os autores sugeriram que vários suplementos poderiam contribuir para o teste positivo para o doping (KAMBER et al., 200 I).

Em artigo de revisão, Yonamine, Garcia e Moreau (2004) consideraram que, a despeito da regulamentação que trata do uso veterinário de esteróides anabólicos, existe a possibilidade do uso ilegal destas substâncias e, consequentemente, a contaminação de resíduos hormonais nos alimentos de origem animal, embora as maiores concentrações fiquem alocadas no fígado, rins e intestino. Os autores apresentam alguns estudos que comprovam tal possibilidade e esclarecem que existem evidências de que o consumo de carne de animais tratados com esteróides anabólicos pode provocar resultados positivos no teste antidoping realizado através da urina.

Cabe destacar que o uso de esteróides anabólico-androgênicos tem como conseqüência o aumento dos níveis de massa muscular e de força e, por isso, tem sido procurada por pessoas desejosas em aumentar o rendimento físico e alterar a estética corporal. A contaminação deliberada de suplementos dietéticos por esteróides está, possivelmente, associada aos interesses dos fabricantes em relação 
aos resultados de seus produtos. Contudo, o uso prolongado destas drogas pode provocar efeitos adversos tais como, infertilidade, alteração no tom de voz, aumento dos pêlos corporais, aumento de secreção das glândulas sebáceas, aumento do tamanho do clitóris, agressividade, diminuição da testosterona plasmática, atrofia testicular, ginecomastia, azoospermia, disfunções hepáticas, alterações cardiovasculares, transtornos psiquiátricos, câncer, entre outros fatores. Entretanto, os pré-hormônios são bem menos estudados e não se sabe ao certo sobre seus efeitos relacionados ao desempenho físico e à saúde. Não obstante, tem sido considerado que seus efeitos à saúde poderiam ser semelhantes aos esteróides, além de potencialmente poderem resultar em testes antidopings positivos (BOYCE, 2003; CHYKA, 2003).

A maioria dos estudos analisados apontou maior risco de contaminação em suplementos para aumento de força e massa muscular, como os pré-hormônais (DHEA, androstenediol, androstenediona, norandrostenediol e norandrostenediona) (VAN DER MERWE; GROBBELAAR, 2005; GEYER et al., 2004; CATLIN et al., 2000). Alguns outros autores observaram a contaminação em tipos diferentes de suplementos como: creatina; aminoácidos e proteínas; e psicoestimulantes (BAUME et al., 2006; KAMBER et al., 200 I ). E ainda em produtos naturais e herbais; diuréticos; repositores energéticos, vitaminas e antioxidantes (KAMBER et al., 200 I).

Ademais, os suplementos mais contaminados apresentaram-se sob a forma de cápsulas (VAN DER MERWE; GROBBELAAR, 2005; GEYER et al., 2004; CATLIN et al., 2000; DE COCK et al., 200 I ; KAMBER et al., 200 I).

\section{CONTAMINAÇÃO POR PSICOESTIMULANTES}

Se a contaminação deliberada de suplementos dietéticos por esteróides pode estar associada aos interesses dos fabricantes em relação aos resultados de seus produtos, o mesmo pode ocorrer em relação à contaminação por psicoestimulantes.

Entre a classe dos psicoestimulantes encontram-se: Efedrina, cafeína, Ma Huang, Guaraná, sinefrina (BAUME et al., 2006).

No estudo conduzido por Kamber et al. (200 I) foi possível observar que nos produtos classificados como "potencializadores mentais" foram encontradas substâncias, como a efedrina e a cafeína, não declaradas nos rótulos. Em algumas marcas a concentração era tão elevada que testes em atletas poderiam ser positivos para o doping.

Nas análises realizadas por Baume et al. (2006) foram encontradas, além de pré-hormônios, a presença de estimulantes na composição de suplementos dietéticos (creatina, BCAAs, pré-hormônios e melhoradores mentais) vendidos pela internet. Foram observadas a presença de efedrina, cafeína e sinefrina, os quais são 
classificados como "potencializadores mentais", na composição química dos produtos. Os autores, porém, não consideraram que houve contaminação porque as substâncias estavam declaradas nos rótulos. No entanto, as mesmas encontravam-se em concentrações mais elevadas do que registradas nos rótulos.

Em um levantamento realizado para detectar a presença de substâncias consideradas ilegais pelo o Comitê Olímpico Internacional e pela Agência Mundial Antidoping em 64 diferentes suplementos nutricionais, observou-se que, do total, 12,5\% dos produtos analisados continham algumas das substâncias proibidas e não estavam declaradas nos rótulos. Entre estas substâncias encontrava-se a efedrina (MARTELLO; FELLI; CHIAROTTI, 2007).

Parr et al. (2008) relatam um caso em que um homem saudável, após comprar um acelerador metabólico pela internet e ingerir dois tabletes, manifestou tremores e apresentou em sua urina $2 \mathrm{ng} / \mathrm{mL}$ de clenbuterol. $O$ mesmo produto consumido foi analisado e revelou a quantidade de 30 microgramas por tablete. Os autores ressaltam que o clenbuterol é classificado como doping e somente deveria ser consumido se fosse legalmente prescrito por médicos. Parr et al. (2008) destacam, ainda, que há um problema recorrente com a fabricação de suplementos, já que fármacos e substâncias dopantes são facilmente encontradas nos produtos comercializados.

Gurley, Gardner e Hubbard (2000) mostraram que quantidades variadas de efedrina foram encontradas em muitos suplementos dietéticos comerciais e mesmo uma variação entre lotes de um mesmo produto foi verificada. Identificaram, também, que em vários produtos o conteúdo de efedrina não estava mencionado no rótulo. Os autores também verificaram, ainda, que pouco mais da metade dos suplementos analisados exibiram discrepâncias (diferenças superiores a 20\%) entre o que estava informado no rótulo e o conteúdo mensurado.

Estudo similar foi desenvolvido por Haller et al. (2004). A quantidade de efedrina, pseudoefedrina, metilefedrina, metilpseudoefedrina e cafeína foram analisadas em 35 suplementos dietéticos comerciais e tiveram seus conteúdos comparados com os rótulos. Os autores encontraram uma variação de 5,97 mg a 29,3 mg de efedrina por porção estudada. Duas marcas não listaram a quantidade de efedrina no rótulo. Além disto, $31 \%$ dos produtos continham quantidade de efedrina superior a $110 \%$ em relação ao informado no rótulo; e cinco de nove produtos variaram a quantidade de efedrina em diferentes lotes analisados.

Pipe e Ayotte (2002) destacam que a efedrina está presente em muitos suplementos nutricionais e são particularmente encontrados em vários produtos destinados aos atletas e praticantes de exercícios físicos, seja propagando a ideia de que pode reduzir o peso corporal ou aumentar o rendimento físico. E como é 
derivada de uma planta é frequentemente considerada como um produto "natural". Contudo, os autores acreditam que o uso desta substância pode ser perigoso e que há evidências de sua associação com a mortalidade por diferentes eventos. Além disto, o uso destes suplementos pode resultar em testes positivos para o doping.

Este entendimento é compartilhado por diversos outros pesquisadores. Green et al. (200 I) concordam que os produtos denominados "potencializadores mentais" ou "aceleradores metabólicos" (Xenadrine, Ripped Fuel e/ou Thermobuterol) com base em efedrina tem sido utilizadas para aumentar o desempenho ou superar a fadiga e favorecer a perda de peso. Entre os efeitos adversos à saúde, Haller e Benowitz (2000) relataram a ocorrência de hipertensão, palpitações, taquicardia, arritmias, infarto do miocárdio, acidente vascular cerebral e convulsões como efeitos mais comuns e sugerem que o uso destas substâncias pode proporcionar um risco à saúde de algumas pessoas, indicando a necessidade de se conhecer melhor as susceptibilidades individuais aos efeitos adversos deste produtos. Yonamine, Garcia e Moreau (2004) igualmente lembram que estimulantes como efedrina, pseudoefedrina, metilefedrina ou norefedrina são incluídos na lista das substâncias proibidas pelo Comitê Olímpico Internacional e pela Agência Mundial Antidoping.

\section{CONCLUSÕES}

Em que pese o uso dos suplementos dietéticos ser bastante difundida entre atletas e praticantes de exercícios físicos com o intuito de prover um complemento à alimentação, alguns pesquisadores entendem que algumas destas substâncias podem não conferir melhorias no desempenho ou à saúde e, mesmo, serem prejudiciais tanto a um aspecto, quanto ao outro.

Foi possível observar que algumas investigações detectaram doses excessivas de ingredientes potencialmente tóxicos, não indicados no rótulo e considerados doping pela Agência Mundial Antidoping (WADA). Entre as substâncias proibidas encontradas nos suplementos estão os esteróides anabólico-androgênicos e os psicoestimulantes.

A despeito de ser possível que a contaminação possa ser decorrente da manipulação inadequada das substâncias químicas, há evidências de que possa ser também fruto da adulteração deliberada do suplemento. Contudo, dois pontos necessitam ser destacados. Primeiro, o risco que a ingestão de determinadas substâncias pode causar à saúde, especialmente, quando o consumidor já apresenta determinados problemas de saúde que podem ser agravados. Segundo, mesmo que sem intenção de usar uma substância proibida (doping), a ingestão inocente destas substâncias não tem sido considerada uma desculpa aceitável e o atleta está susceptível a sanções, 
uma vez que se tem em conta o "princípio da responsabilidade", presente no inciso 2. I . I do artigo 2. I do código da Agência Mundial Antidoping.

Neste sentido, é possível alegar que o uso de suplementos dietéticos por praticantes de atividades físico-esportivas, competitivas ou não, carece de maior segurança e deve ser realizado com muito critério. É importante ressaltar que a indicação ou prescrição de suplementos dietéticos é atividade de nutricionistas ou médicos e, portanto, é necessário que a população alvo (atletas e praticantes de atividade física) seja conscientizada dos riscos do consumo indevido desses produtos.

Além disso, há necessidade de se realizar mais estudos em produtos brasileiros, para que a indicação dos suplementos seja feita de forma mais consciente e segura por parte desses profissionais.

Contamination of dietary supplements used for sport practice: a review of literature

ABSTRACT: The purpose of this study was to review the literature about the contamination of dietary supplements by substances considered harmful to health or doping to athletes. Scientific articles indexed in the databases Medline and Scielo were searched. It was observed that some investigations had detected excessive doses of potentially toxic not labeled ingredients that are considered doping. Among the illegal substances found in some supplements there are the anabolic-androgenic steroids and stimulants. It can be concluded that the use of dietary supplements by practitioners of physical activities, competitive or not, needs more security and should be done carefully.

KEYWORDS: Dietary supplements; anabolic-androgenic steroids; contamination; doping.

\section{Contaminación de suplementos dietéticos usados para la práctica deportiva: una revisión de literatura}

RESUMEN: El objetivo del presente estudio fue revisar la literatura que trata de la contaminación de los suplementos alimentares por sustancias consideradas nocivas a la salud o doping para los atletas. Para eso, fueron revisados artículos científicos indexados en las bases de datos Medline y Scielo. Fue posible observar que algunas investigaciones detectaron dosis excesivas de ingredientes potencialmente tóxicos, no indicados en el rótulo y considerados doping. Entre las sustancias prohibidas encontradas en algunos suplementos están los esteroides anabólico-androgénicos y los psicoestimulantes. En este sentido, es posible alegar que el uso de suplementos dietéticos por practicantes de actividades físico-deportivas, competitivas o no, carece de mayor seguridad y debe ser realizado con mucho criterio.

PALABRAS CLAVE: Suplementos dietéticos; esteroides anabólico-androgénicos; contaminación; doping. 


\section{REFERÊNCIAS}

AMERICAN DIETETIC ASSOCIATION, DIETITIANS OF CANADA AND AMERICAN COLLEGE OF SPORTS MEDICINE. Position of American Dietetic Association, Dietitians of Canada and American College of Sports Medicine: nutrition and athletic performance. Journal of the American Dietetic Association, Chicago, v. 100, n. 12, p. I543-56, dec. 2000.

BARROS NETO, T. L. A controvérsia dos agentes ergogênicos: estamos subestimando os efeitos naturais da atividade física?. Arquivos Brasileiros de Endocrinologia \& Metabologia, São Paulo, v. 45, n. 2, p. 121 -122, mar/abr. 2001.

BAUME, N. et al. Research of stimulants and anabolic steroids in dietary supplements. Scandinavian Journal of Medicine \& Science in Sports, Oxford, v. 16, n. I, p. 4I-8, feb. 2006.

BOYCE, E.G. Use and effectiveness of performance-enhancing substances. Journal of Pharmacy Practice, New York, v. 16, n. I, p. 22-36, feb. 2003.

BRAUN, H. et al. Dietary supplement use among elite young German athletes. International Journal of Sport Nutrition and Exercise Metabolism, Champaign, v. 19, n. I, p. 97-109, feb. 2009.

CATLIN, D. H. et al. Trace contamination of over-the-counter androstenedione and positive urine test results for a nandrolone metabolite. The Journal of the American Medical Associatian, Chicago, v. 284, n. 20, p. 2618-2621, nov. 2000.

CHYKA, P. A. Health risks of selected performance-enhancing drugs. Journal of Pharmacy Practice, New York, v. 16, n. I, p. 37-44, feb. 2003.

DE COCK, K. J. et al. Detection and determination of anabolic steroids in nutritional supplements. Journal of Pharmaceutical and Biomedical Analysis, London, v. 25, n. 5-6, p. 843-852, jul. 2001 .

GEYER, H. et al. Analysis of non-hormonal nutritional supplements for anabolic-androgenic steroids - results of an international study. International Journal of Sports Medicine, Stuttgart, v. 25, n. 2, p. 124-129, feb 2004.

GREEN, G. A. et al. NCAA study of substance use and abuse habits of college student-athletes. Clinical Journal of Sport Medicine, Philadelphia, v. II, n. I, p. 5I-56, jan. 200I.

GURLEY, B. J.; GARDNER, S. F.; HUBBARD, M. A. Content versus label claims in ephedracontaining dietary supplements. American Journal of Health-System Pharmacy, Marylan, v. 57, n. 10, p. 963-969, may 2000.

HALLER, C. A.; BENOWITZ, N. L. Adverse cardiovascular and central nervous system events associated with dietary supplements containing Ephedra alkaloids. New England Journal of Medicine, Massachusetts, v. 343, n. 25, p. 1833-1838, dec. 2000. 
HALLER, C. A. et al. Concentrations of ephedra alkaloids and caffeine in commercial dietary supplements. Journal of Analytical Toxicology, Danvers, v. 28, n. 3, p. I45- I 5 , apr. 2004.

JUHN, M. S. Popular sports supplements and ergogenic aids. Sports Medicine, London, v. 33, n. 12, p. 921-939, dec. 2003.

KAMBER, M. et al. Nutritional supplements as a source for positive doping cases?. International Journal of Sport Nutrition and Exercise Metabolism, Champaign, v. I I, n. 2, p. 258-263, jun. 2001 .

KAVA, R. et al. Dietary supplement safety information in magazines popular among older readers. Journal of Health Communication, Washington, v. 7, n. I, p. I3-23, jan./feb. 2002.

KRISTIANSEN, M. et al. Dietary supplement use by varsity athletes at a Canadian University. International Journal of Sport Nutrition and Exercise Metabolism, Champaign, v. I 5, n. 2, p. 195-2 10, apr. 2005.

MARTELLO, S.; FELLI, M.; CHIAROTTI, M. Survey of nutritional supplements for selected illegal anabolic steroids and ephedrine using LC-MS/MS and GC-MS methods, respectively. Food Additives \& Contaminants, Abingdon, v. 24, n. 3, p. 258-65, mar. 2007.

MAUGHAN, R. J. Contamination of dietary supplements and positive drug tests in sport. Journal of Sports Sciences, Abingdon, v. 23, n. 9, p. 883-889, sep. 2005.

; KING, D. S.; LEA, T. Dietary supplements. Journal of Sports Sciences, Abingdon, v. 22, n. I, p. 95- I 13, jan. 2004.

MORRISON, L. J.; CIZIS, F.; SHORTER, B. Prevalent use of dietary supplements among people who exercise at a Commercial Gym. International Journal of Sport Nutrition and Exercise Metabolism, Champaign, v. I4, n. 4, p. 48I-492, aug. 2004.

PARR, M. K. et al. Clenbuterol marketed as dietary supplement. Biomedical Chromatography, Malden, v. 22, n. 3, p. 298-300, mars 2008.

PEREIRA, R. F.; LAJOLO, F. M.; HIRSCHBRUCH, M. D. Consumo de suplementos por alunos de academias de ginástica em São Paulo. Revista de Nutrição, Campinas, v. 16, n. 3, p. 265-272, jul./set. 2003.

PIGNARRE, P. O que é o medicamento: um objeto estranho entre ciência, mercado e sociedade. São Paulo: Editora 34, 1999.

PIPE, A.; AYOTTE, C. Nutritional Supplements and Doping. Clinical Journal of Sport Medicine, Philadelphia, v. 12, n. 4, p. 245-49, jul. 2002.

SANTOS, A. M. O mundo anabólico: análise do uso de esteróides anabólicos nos esportes. Barueri: Manole, 2003.

SILVER, M. D. Use of ergogenic aids by athletes. Journal of the American Academy of Orthopaedic Surgeons, Birmingham, v. 9, n. I, p. 6I-70, jan./feb. 200।. 
VAN DER MERWE, P. J.; GROBBELAAR, E. Unintentional doping through the use of contaminated nutritional supplements. South African Medical Journal, Pretoria, v. 95, n. 7, p. 5।0-5। I, jul. 2005.

WORLD ANTI-DOPING AGENCY. World anti-doping code. Montreal: World Anti-Doping Agency, 2003.

WORLD ANTI-DOPING AGENCY. Athlete guide. Montreal: World Anti-Doping Agency, 2005.

WORLD ANTI-DOPING AGENCY. World anti-doping code: the 2008 prohibited list international standard. Montreal: World Anti-Doping Agency, 2007.

YONAMINE, M.; GARCIA, P. R.; MOREAU, R. L. M. Non-intencional doping in sports. Sports Medicine, London, v. 34, n. II, p. 697-704, Nov. 2004.

Recebido: 19 maio 2010

Aprovado: 10 nov. 2010

Endereço para correspondência:

Alexandre Palma

Universidade Federal do Rio de Janeiro

Escola de Educação Física e Desportos

Av. Carlos Chagas Filho, 540,

Edifício da Educação Física, Cidade Universitária

2194 I-599, Rio de Janeiro, RJ, Brasil 